\title{
FINE CONVERGENCE AND ADMISSIBLE CONVERGENCE FOR SYMMETRIC SPACES OF RANK ONE
}

BY

\author{
ADAM KORÁNYI ${ }^{1}$ AND J. C. TAYLOR ${ }^{2}$
}

\begin{abstract}
The connections between fine convergence in the sense of potential theory and admissible convergence to the boundary for quotients of eigenfunctions of the Laplace-Beltrami operator are investigated. This leads to a version of the local Fatou theorem on symmetric spaces of rank one which is considerably stronger than previously known results.

The appendix establishes the relationship between harmonic measures on the intersection of the Martin boundaries of a domain and a subdomain.
\end{abstract}

Introduction. The extensions of the classical theorem of Fatou on the unit disc to noncompact symmetric spaces have by now produced a considerable literature (cf. [17] for a recent survey).

Earlier extensions of the Fatou theorem were developed for the classical harmonic functions on a Euclidean half-space. In this context stronger results have been obtained than in the symmetric space case. The best one of these is the local version of Fatou's theorem, due to Calderón [3] and improved upon by Carleson [4]. Let $E$ be a subset of $\left\{x_{n}=0\right\} \subset \mathbf{R}^{n}$ whose complement has measure zero and for each $x \in E$ let $C(x)$ be a cone with vertex at $x$ lying in $\left\{x_{n}>0\right\}$. If $u$ is a harmonic function on $W=\cup\{C(x) \mid x \in E\}$ with a one-sided bound on each cone $C(x)$ then $u$ has a nontangential limit a.e. on the hyperplane $\left\{x_{n}=0\right\}$.

Brelot and Doob [2] improved this result further by proving it for quotients $u / h$ with $u$ harmonic on $W, h$ positive harmonic on $\left\{x_{n}>0\right\}$ and $u / h$ with a one-sided bound on each $C(x)$. Their proof used the theory of fine convergence at the Martin boundary and they established the equivalence modulo null sets of nontangential convergence and fine convergence for quotients $u / h$ of harmonic functions.

In the present paper the methods of [2] are used on a symmetric space of rank one to prove for such a space the analogue of the local Fatou theorem for quotients $u / h$, where $u$ and $h$ are solutions of $\Delta v=c v, c>-|\rho|^{2}$ (see below for the definition of $\rho$ ). In [19] this result is proved for $h=1, u$ bounded and $c=0$ and in [21], for globally defined $u$, the same result is obtained for a particular $h$ (the spherical function).

Received by the editors November 5, 1979.

AMS (MOS) subject classifications (1970). Primary 31B25, 31D05, 53C35.

Key words and phrases. Rank one symmetric space, admissible convergence, eigenfunction of Laplace-Beltrami operator, fine convergence, Martin boundary, local Fatou theorem.

${ }^{1}$ Partially supported by the National Science Foundation.

${ }^{2}$ Materially supported by NSERC operating grant No. A-3108.

(c) 1981 American Mathematical Society 0002-9947/81/0000-0009/\$04.25 
Since the theory of fine convergence can be discussed in terms of the associated diffusion (see Doob [8] and Föllmer [11]) it would of course be possible to obtain these results with probabilistic arguments. It is to be noted that for the unit ball in $\mathbf{C}^{n}$ and $c=0$ Debiard [7] showed for a globally defined harmonic function $u$ that fine convergence implies admissible convergence.

In this article no use is made of the associated diffusion. For a detailed and more elementary treatment of these questions in the case of the unit ball in $\mathbf{C}^{n}$ and $c=0$ see [25].

Let $f(s)$ and $g(s)$ be two functions defined for $s$ near $s_{0}$. The notations $f(s) \sim g(s)$ and $f(s) \approx g(s)$ indicate respectively that $f(s) / g(s)$ has a nonzero limit as $s \rightarrow s_{0}$ and that there is a constant $C$ with $1 / C \leqslant f(s) / g(s)<C$ for $s$ close enough to $s_{0}$.

An arbitrary constant will be denoted by $C$ or $c$ and may change its value from one use to another.

Finally, the complement of a set $E$ will be denoted by $E^{c}$.

\section{Preliminaries. Fine limits and symmetric spaces.}

$1^{\circ}$. For the reader's convenience a brief introduction to potential theory and fine limits is given below. Let $X$ be a connected, metrizable $C^{\infty}$-manifold of dimension $d$ and let $\mathcal{H}$ denote the sheaf of $C^{2}$-functions $u$ with $L u=0$ where, in local coordinates, $L$ is a second order elliptic operator with (say) smooth coefficients. Then $\mathcal{H}$ satisfies the axioms of Brelot [14]. A $C^{2}$-function $u$ such that $L u=0$ will be said to be harmonic.

Assume that a Green function $G$ exists, i.e., $G: X \times X \rightarrow[0,+\infty]$ is lower semicontinuous, continuous off the diagonal and such that $L G_{y}=0$ on $X \backslash\{y\}$ (where $G_{y}(x)=G(x, y)$ ), $G_{y}(x) \sim\|x-y\|^{2-d}$ if $d>2$ and $\sim-\log \|x-y\|$ if $d=2$ and majorizes no positive harmonic functions other than 0 .

Fix a point $a \in X$ and set $K(x, y)=1$ if $x=y=a$ and $=G(x, y) / G(a, y)$ otherwise. The Martin compactification $\hat{X}$ of $X$ is the compactification to which all the functions $y \leadsto K(x, y)$ extend continuously in such a way that their extensions separate the points of $\Delta=\hat{X} \backslash X$ (cf. [24]). For $b \in \Delta$ set $K(x, b)=\lim _{y \rightarrow b} K(x, y)$. Then $x \leadsto K(x, b)=K_{b}(x)$ is harmonic, the set $\Delta_{1}=\left\{b \in \Delta \mid K_{b}\right.$ is minimal $\}$ is a Borel set (even a $G_{\delta}$ ) and each positive harmonic function $u$ can be written uniquely as $u(x)=\int K(x, b) \mu(d b)$ with $\mu$ carried by $\Delta_{1}$ (the representing measure). Note that a positive harmonic function $h$ is said to be minimal if $0 \leqslant k \leqslant h$ and $k$ harmonic implies $k=\lambda h, \lambda \in[0,1]$.

If $O \subset X$ is open and relatively compact then to each $f \in C(\partial O)$ corresponds a harmonic function $H f$ on $O$ (solution of the Dirichlet problem). The functional $f \leadsto H f(x)$ is equal to $\int f d \mu_{x}^{O}$ for a unique so-called harmonic measure $\mu_{x}^{O}, x \in O$. The nonnegative lower semicontinuous functions $u$ with $u(x)>\int u d \mu_{x}^{O}, \forall O$ open relatively compact and $\forall x \in O$ are said to be superharmonic (unless $u \equiv+\infty$ ). For any set $E \subset X$ and $u$ superharmonic the réduite of $u$ on $E$ is defined to be $R_{E} u=\inf \{v \mid v \geqslant u$ on $E, v$ superharmonic $\}$. Its lower-semicontinuous regularization, denoted by $\hat{R}_{E} u$, is superharmonic.

A set $E$ is said to be (minimally) thin at $b \in \Delta_{1}$ if $R_{E} K_{b} \neq K_{b}$. This is the case if and only if $\hat{R}_{E} K_{b}$ is a superharmonic function that majorizes no positive harmonic 
function other than zero [12]. Consequently, if $E_{1}$ and $E_{2}$ are thin at $b$ it follows from the obvious inequality $R_{E_{1} \cup E_{2}} K_{b} \leqslant R_{E_{1}} K_{b}+R_{E_{2}} K_{b}$ that $E_{1} \cup E_{2}$ is also thin at $b$. Hence, $\mathscr{F}(b)=\left\{E \mid E^{c}\right.$ is thin at $\left.b\right\}$ is a filter.

Definition 0.1. $\mathscr{F}(b)$ is said to be the fine filter at $b$. A function $f$ converges finely $a t b$ or has a fine limit $a t b$ if it converges along $\mathscr{F}(b)$.

It is known that for any neighborhood $U$ of $b$ in $\hat{X}$ the set $(U \cap X)^{c}$ is thin at $b$ (due to Martin [20] in the classical case; his argument applies whenever there is a Green function). Hence, $\mathscr{F}(b)$ contains the filter of relative neighbourhoods of $b$ in $X$.

Let $u, h$ be two positive harmonic functions on $X$. The following result is known as the Fatou-Naïm-Doob limit theorem ([9], [12], [22]).

THEOREM 0.2. Let $\mu$ and $\nu$ be the representing measures for $u$ and $h$ respectively. Then $u / h$ converges finely to $d \mu / d \nu$ at $\nu$-almost every minimal point.

$2^{\circ}$. From now on $X$ will denote a symmetric space of noncompact type of rank one. Let $G$ denote its connected isometry group and denote by $K$ the isotropy group of a fixed point $o \in X$. The Cartan and Iwasawa decompositions of the Lie algebra $g$ of $G$ will be denoted by $\mathfrak{f} \mathfrak{p}$ and $\mathfrak{f}+\mathfrak{a}+\mathfrak{n}$. Let $2 \rho$ denote the sum of the positive roots with multiplicities counted i.e. if $\alpha$ is the simple positive root, $2 \rho=(p+2 q) \alpha$ where $p$ (resp. $q)$ is the dimension of the eigenspace corresponding to $\alpha$ (resp. $2 \alpha)$. Choose $H \in$ a such that $\alpha(H)=1$. Then $2 \rho(H)=p+2 q$.

Let $\Delta$ denote the Laplace-Beltrami operator on $X$ associated with the $G$-invariant Riemannian metric on $X$. Denote by $c$ any number $>-|\rho|^{2}$, where $|\rho|$ is the length of $\rho$ as an element of the dual of $a$ when $a$ is equipped with the inner product coming from the identification of $\mathfrak{p}$ with the tangent space at $o$ (the metric on $X$ is normalized so that $\|H\|=1$ ). In other words, $|\rho|=p / 2+q$.

For the remainder of this article a function $u$ will be said to be harmonic if $\Delta u=c u$. The choice of the lower bound for $c$ ensures that there exists a positive harmonic function on $X$ and that a Green function exists [15].

Since $X$ is of rank one, the Martin boundary can be identified with the Furstenberg boundary $G / M A N \cong K / M$ where the Lie algebra $\mathfrak{m}$ of $M$ is the centralizer of $a$ in $g$ and the Poisson kernel $K(x, \dot{k})=\exp (-1)(\rho+\lambda) H\left(g^{-1} k\right)$ where $\lambda=\left(\sqrt{|\rho|^{2}+c}\right) \alpha, x=g \cdot o$ and $H\left(g^{-1} k\right)$ is the logarithm of the $A$-component of $g^{-1} k$ in its Iwasawa decomposition $G=K A N$.

Let $B(x ; r)$ denote the closed geodesic ball about $x$ of radius $r$.

Definition 0.3. For $r>0$, the set $A(\dot{k} ; r)=\{k \exp t H \cdot x \mid t>0, x \in B(o ; r)\}$ will be called an admissible region at $\dot{k}$ of width $r$. If in addition $t \geqslant T$, for some $T$, the region will be said to be truncated and will be denoted by $A^{T}(\dot{k} ; r)$. A function $f$ is said to converge admissibly at $\dot{k}$ to $l$ if for each width $r>0$ and $\varepsilon>0$ there exists $T=T(\varepsilon ; r)$ such that $|f(x)-l|<\varepsilon$ for $x \in A^{T}(\dot{k} ; r)$.

REMARK. As pointed out in [17], $A(k ; r)$ is the set of points whose distance from the geodesic line $t \leadsto k \exp t H \cdot o$ is at most $r$.

Returning to the Iwasawa decomposition, let $\overline{\mathfrak{n}}$ denote the image of $\mathfrak{n}$ under the Cartan involution. If $\bar{N}$ and $A$ denote the analytic subgroups of $G$ corresponding to 
$\overline{\mathrm{n}}$ and a then $\bar{N} A$ is a group (since $A$ normalizes $\bar{N}$ ) and every element $x \in X$ has a unique expression as $x=\bar{n} a \cdot o=\bar{n} \exp t H \cdot o$ with $\bar{n} \in \bar{N}, a \in A$ and $t \in \mathbf{R}$.

The lemma of Bruhat and Harish-Chandra [26, Theorem 1.2.3.1] implies that the action of the nilpotent group $\bar{N}$ on $G / M A N \cong K / M$ is such that the orbit of some point $b$ is an open dense set which can be identified with $\bar{N}$ since $\bar{n} \leadsto \bar{n} \cdot b$ is injective [17, Lemma 2.3]. Let $e$ denote $M A N$ and identify $\bar{N}$ with $\bar{N} \cdot e$.

Choose some Haar measure $d \bar{n}$ on $\bar{N}$ and a smooth homogeneous gauge, i.e., a $C^{\infty}$-function ||$: \bar{N} \backslash\{e\} \leadsto \mathbf{R}^{+}$such that $\left|\bar{n}^{\exp t H \cdot o}\right|=e^{-t}|\bar{n}|$ and $\left|\bar{n}^{-1}\right|=|\bar{n}|$, where $\bar{n}^{a}=a \bar{n} a^{-1}$. Let $k \geqslant 1$ be such that $\left|\bar{n} \bar{n}^{\prime}\right|<k\left(|\bar{n}|+\left|\bar{n}^{\prime}\right|\right)$ (cf. [19]). For $r>0$ and $\bar{n} \in N$ set

$$
\begin{aligned}
\Gamma^{T}(\bar{n} ; r) & =\left\{\bar{n}(\exp t H) \bar{n}^{\prime} \cdot o|| \bar{n}^{\prime} \mid<r, t>T\right\} \\
& =\left\{\bar{n} \bar{n}^{\prime}(\exp t H) \cdot o|| \bar{n}^{\prime} \mid<r e^{-t}, t>T\right\} .
\end{aligned}
$$

Lemma 0.4. (SEe [17].) Let $r, T>0$. Then there exist $r^{\prime}, T^{\prime}$ such that $A^{T^{\prime}}\left(\bar{n} ; r^{\prime}\right) \subset$ $\Gamma^{T}(\bar{n} ; r)$ and $r^{\prime \prime}, T^{\prime \prime}$ such that $\Gamma^{T^{\prime \prime}}\left(\bar{n} ; r^{\prime \prime}\right) \subset A^{T}(\bar{n} ; r)$.

As a result, admissible convergence can also be defined and discussed in terms of the regions $\Gamma^{T}(\bar{n} ; r)$.

1. Potential theory for $\Delta u=c u$. It follows from [13, pp. 381-382] that the area $A(t)$ of the geodesic sphere of radius $t$ and centre $o$ is $C \sinh ^{p} t \sinh ^{q}(2 t)$. Consequently by [13, p. 445], the radial form of the equation $\Delta u=c u$ is given by $L w=c w$, where

$$
L w=w^{\prime \prime}(t)+a(t) w^{\prime}(t) \quad \text { with } a(t)=\{\log A(t)\}^{\prime}=(p+q) \operatorname{coth} t+q \tanh t
$$

and $u(x)=w(t)$ if $t$ is the geodesic distance of $x$ from $o$.

The coefficient $a(t)$ converges to $p+2 q$ at $+\infty$ and so the substitution $s=e^{-t}$, $w(t)=\varphi(s)$ gives $L w(t)=M \varphi(s)=s^{2} \varphi^{\prime \prime}(s)+s(1-a(t)) \varphi^{\prime}(s)-c \varphi(s)$ with 1 $a(t)=b(s)$ analytic for $|s|<1$ and $b(0)=1-(p+2 q)$. The indicial equation for $M \varphi=0$ is $r(r-1)+r(1-(p+2 q))-c=r^{2}-r(p+2 q)-c=0$ and the classical method of Frobenius (cf. [5]) shows that there is a fundamental set $\left\{w_{1}, w_{2}\right\}$ of solutions to $L w-c w=0$ on $(0,+\infty)$ with the following properties:

(1) if $c>-|\rho|^{2}=-(p / 2+q)^{2}$ and $r_{1}=|\rho|-\sqrt{|\rho|^{2}+c}, r_{2}=|\rho|+\sqrt{|\rho|^{2}+c}$ are the roots of the indicial equation then $w_{i}(t) \sim e^{-r_{i} t}$ and $w_{i}^{\prime}(t) \sim r_{i} e^{-r_{i} t}$ at $+\infty$ $(i=1,2)$; and

(2) if $c=-|\rho|^{2}$ then $w_{1}(t) \sim t e^{-|\rho| t}, w_{1}^{\prime}(t) \sim(-|\rho|) t e^{-|\rho| t}$ and $w_{2}(t) \sim e^{-|\rho| t}, w_{2}^{\prime}(t)$ $\sim(-|\rho|) e^{-|\rho| t}$ at $+\infty$.

According to Karpelevič [15], $\Delta u-c u=0$ has a nontrivial positive solution if and only if $c \geqslant-|\rho|^{2}$. By averaging over $K$ it is clear that $\Delta u-c u=0$ has therefore a positive spherical solution $\phi$ for $c>-|\rho|^{2}$. Furthermore, $\phi$ is well known to be unique (up to a scalar) [13].

If $|\rho|^{2}$ is written as $\rho^{2}$ and $-c=\lambda^{2}+\rho^{2} \leqslant \rho^{2}$ then $\lambda$ is purely imaginary and can be assumed to be in $i \mathbf{R}^{+}$. It is clear that up to constants, $w_{2}(t)$ and $\phi$ are the functions $\Phi_{\lambda}$ and $\phi_{\lambda}$ respectively of [10].

Proposition 1.1. If $\phi=a_{1} w_{1}+a_{2} w_{2}$ then $a_{1} \neq 0$. Hence, $\phi(t) \sim w_{1}(t)$. 
Proof. The first statement is due to Harish-Chandra. A simple proof of this fact may be found in [10]. More precisely, Lemma 8 of [10] shows that $c^{-}(\lambda) \neq 0$ for $\lambda \in i \mathbf{R}^{+}$and so as $\phi_{\lambda}(t)=c^{+}(\lambda) \Phi_{\lambda}(t)+c^{-}(\lambda) \Phi_{-\lambda}(t)$ the asymptotic behaviour of $\phi_{\lambda}(t)$ is that of $w_{1}(t)$, i.e., $a_{1} \neq 0$.

Proposition 1.2. If $\Delta u=c u$ admits a positive solution then the equation has a Green function. Furthermore, if $G$ is a Green function with pole at o then

$$
G(t) \sim \exp (-t)\left\{|\rho|+\sqrt{|\rho|^{2}+c}\right\}=\exp \left(-r_{2} t\right) .
$$

Proof. Assume $u$ is radial and define $v$ by $u=v \phi$. Then (by "reduction of order") $L u=c u$ if and only if $v^{\prime \prime}+\left[\left(2 \phi^{\prime}\right) / \phi+a\right] v^{\prime}=0$. The general form of $v$ is $v(t)=v(1)+A v_{0}(t)=v(1)+A \int_{1}^{t} \exp (-B(s)) d s$, where $B(s)=$ $\int_{1}^{s}\left[\left(\left(2 \phi^{\prime}(x)\right) / \phi(x)\right)+a(x)\right] d x$. The fact that $\phi \sim w_{1}$ and so is not a multiple of $w_{2}$ implies (by explicit computation) that $\exp (-B(s))$ is dominated at $+\infty$ by an integrable function (in fact by a multiple of $1 / s^{2}$ ).

Consequently, if $A=-1$ the constant $v(1)$ can be chosen so that $\lim _{t \rightarrow+\infty} v(t)$ $=0$. Since $\lim _{t \rightarrow 0^{+}} v_{0}(t)=-\infty$ (again, explicit computation shows it to be $\sim-t^{(n-2)}$ where $n$ is the real dimension of $X$ if $n>3$ ) it follows that the resulting function $G=v \phi$ is a Green function with pole at $o$.

Let $G=c_{1} w_{1}+c_{2} w_{2}$. Then $v=G / \phi=G /\left[a_{1} w_{1}+a_{2} w_{2}\right]$ converges to zero as $t \rightarrow \infty$ if and only if $c_{1}=0$.

REMARK. In [15] Karpelevič showed the existence of the Green function for $c \geqslant|\rho|^{2}$ by using the associated heat equation and an exhaustion of $X$ by regular relatively compact domains. This result and the asymptotic behaviour of $\phi$ show immediately that $G(t) \sim \exp \left(-r_{2} t\right)$.

Proposition 1.3 (HARNACK's INEQuality). Let $0<r_{1}<r_{2}$. Then there is a constant $C=C\left(r_{1}, r_{2}\right)$ such that if $u$ is a positive harmonic function (i.e., $\Delta u=c u$ ) on the geodesic ball $B\left(x ; r_{2}\right)$ about $x$ of radius $r_{2}$ then

for all $y \in B\left(x ; r_{1}\right)$.

$$
(1 / C) u(y) \leqslant u(x) \leqslant C u(y)
$$

The constant $C$ may be taken arbitrarily close to 1 providing $r_{1}$ is small enough.

Proof. Since the operator $\Delta-c I$ is $G$-invariant this result is a special case of Serrin's Harnack inequality [23] as long as $c \geqslant 0$.

Assume $c<0$ and let $\phi$ be a positive spherical solution of $\Delta u-c u=0$. If $t=t(x)$ is the geodesic distance of $x$ from 0 then by abuse of notation $\phi(x)=\phi(t)$. Set $u=v \phi$. Then $\Delta u-c u=0$ if and only if $M v=0$ where $M v=\Delta v+$ $2(\log \phi)^{\prime} X v$ and $X=\operatorname{grad} t$ (the intrinsic gradient of $\left.t\right)$.

Consider positive solutions $v$ on $B\left(o ; r_{2}\right)=B$ of $M v=0$. Serrin's Harnack inequality [23] applies to give a constant $C$ such that $(1 / C) v(y) \leqslant v(o)<C v(y)$ for all $y \in B\left(o ; r_{1}\right)$.

Now let $\Delta u=c u, u \geqslant 0$ on $B\left(x ; r_{2}\right)$ and let $g \cdot o=x$. Set $w(y)=u(g \cdot y)$ for $y \in B$. Then $(\Delta u)(g \cdot y)-c u(g \cdot y)=(\Delta w)(y)-c w(y)$. Define $v(y)=$ $w(y) / \phi(y)$. Then since $v$ satisfies a Harnack inequality on $B$ it follows that $w$ satisfies a Harnack inequality on $B$. In other words, $u$ satisfies (*) on $B\left(x ; r_{2}\right)$. 
Consider a system of coordinates at $o$. The Schauder estimates (cf. [6]) and (*) show that for the positive harmonic functions $u$ with $u(o)=1$ the euclidean gradient $\nabla u$ (relative to these coordinates) is uniformly bounded on a neighbourhood of $o$. Hence, the functions $u$ are equicontinuous at $o$. Since $\Delta-c I$ is $G$-invariant the same result holds at any point $x$. This proves the last assertion.

REMARK. This result partially extends Lemma (2.1) in [21].

2. Fine convergence implies admissible convergence. The arguments used in [2] to prove that fine convergence implies nontangential convergence on a half-space can be applied to rank one symmetric spaces because of the following key result.

Proposition 2.1. Let $B=B\left(x ; r^{\prime}\right)$ and let $t=d(o, x)$. Then $R_{B} 1(o) \sim e^{-r_{2} t}$ as $t \rightarrow+\infty$.

If in addition $x \in A(b ; r)$ then there exists a constant $c>0$ such that $R_{B} K_{b}(o)>$ $c$ for $t$ large enough.

Proof. Let $g \in G$ be such that $g \cdot x=o$. Set $\bar{x}=g \cdot o$. Then, $R_{B} 1(o)=R_{g \cdot B} 1(\bar{x})$ $=G(\bar{x}) / G(r)=G(x) / G(r) \sim e^{-r_{2} t}$.

If $x \in A(b ; r)$ then $x=k \exp s H \cdot x^{\prime}$ for some $x^{\prime} \in B(o ; r)$. Also, if $y=$ $k \exp s H \cdot o$ then $|s-t| \leqslant r$ as $s=d(o, y)$. Hence, $R_{B} 1(o) \approx e^{-r_{2} s}$. Furthermore, it is well known (cf. [15] or [26]) that $K_{b}(y)=e^{r_{2} s}$. Since $B \subset B\left(y ; r+r^{\prime}\right)$, it follows that $K_{b} \approx K_{b}(y)=e^{r_{2} s}$ on $B$. The result follows as $R_{B} K_{b}(o)>C R_{B} 1(o)$ if $K_{b}>C$ on $B$.

RemarK. The essential point here is that $K_{b}(x) G(x) \approx 1$ on any fixed $A(b ; r)$. This can be easily verified given Harnack's inequality and the asymptotic behaviour of $G$ along the geodesic $t \leadsto k \exp t H \cdot o$.

THEOREM 2.2. Let $u, h$ be two positive harmonic functions on $X$ (i.e., solutions of $\left.\Delta v=c v, c \geqslant-|\rho|^{2}\right)$. Assume $u / h$ converges finely to $l$ at $b \in K / M$. Then $u / h$ converges admissibly to $l$ at $b$.

Proof (CF. PROOF OF THÉorème 3 IN [2]). It will be enough to show that if $r>0$ and if $\left(x_{n}\right) \subset A(b ; r)$ converges to $b$ and is such that $u\left(x_{n}\right) / h\left(x_{n}\right) \rightarrow l_{1}$ then $l_{1}=l$, i.e., $l$ is the only admissible cluster value.

Assume for some $r>0$ that there exists $\left(x_{n}\right) \subset A(b ; r)$ converging to $b$ with $u\left(x_{n}\right) / h\left(x_{n}\right) \rightarrow l_{1}$.

Let $\varepsilon>0$. By Proposition 1.3 there exists $r^{\prime}>0$ such that $\left|u(x) / h(x)-l_{1}\right|<\varepsilon$ for all $x \in B\left(x_{n} ; r^{\prime}\right)=B_{n}$ providing $n \geqslant N$. Let $E=\cup_{n>N} B_{n}$. It will suffice to show that $E$ is not thin at $b$ since then $E \cap F \neq \varnothing$ for all $F \in \mathscr{F}(b)$ and so $l_{1}=l$.

To prove this first note that $\lim _{m \rightarrow \infty} R_{E_{m}} K_{b}=h$ is nonnegative and harmonic on $X$, where $E_{m}=\cup_{n \geqslant m} B_{n}$. Also, $R_{E_{m}} K_{b}(o) \geqslant R_{B_{m}} K_{b}(o)$. Hence, by Proposition 2.1 there exists a constant $c>0$ with $h(0) \geqslant c$. Since $h$ is a harmonic minorant of $R_{E} K_{b}$ and hence of $\hat{R}_{E} K_{b}$ it follows that $E$ is not thin at $b$.

Remarks. (1) In [2] Brelot and Doob proved a stronger result: semifine convergence of $u / h$ implies nontangential convergence. The semifine filter $\delta(b)$ at $b$ is $\left\{E \mid E^{c}\right.$ is semithin at $\left.b\right\}$ where $E$ is semithin at $b$ if $\lim _{\lambda \rightarrow \infty} \lambda R_{E \cap N_{\lambda}} G=0$, $N_{\lambda}=\left\{K_{b} \geqslant \lambda G\right\}$. Note that $\mathcal{S}(b) \supset \mathscr{F}(b)$. 
This result extends to the present situation. One has only to prove that $E$ in the preceding proof is not semithin: taking $\lambda_{n}=G\left(x_{n}\right)^{-2}$ the remark following Proposition 2.1 implies that $E \cap N_{\lambda_{n}} \supset B_{n}$ for large $n$; the first assertion of Proposition 2.1 implies $c^{\prime} \leqslant \lambda_{n} R_{B_{n}} G(o) \leqslant \lambda_{n} R_{E \cap N_{\lambda_{n}}} G(o)$.

(2) In the case of the upper half-space nontangential convergence and semifine convergence are equivalent for quotients $u / h$ of positive harmonic functions. In [25] the corresponding result is proved for the unit ball in $\mathbf{C}^{\boldsymbol{n}}$. It is also possible to prove the following result by adapting the arguments of Brelot and Doob from [2].

THEOREM 2.3. Let $u, h$ be positive harmonic functions on $X$ (with $c>-|\rho|^{2}$ ). Then $u / h$ converges admissibly at $b \in K / M$ if and only if $u / h$ has a semifine limit at $b$.

The key lemma in the proof states that $E$ is semithin at $b$ if $\lim _{x \rightarrow b} K_{b}(x) G(x) 1_{E}(x)=0$.

Since it is always true that $\mathcal{S}(b) \supset \mathscr{F}(b)$ Theorem 2.3 implies Theorem 2.2.

(3) It will be shown in $\S 4$ that, except for a set of $d \bar{n}$ measure zero, if a function $f$ has an admissible limit at $\bar{n} \in \bar{N}$ then $f$ has a fine limit at $\bar{n}$.

(4) In the proof of Theorem 2.2 it is not essential that $u$ and $h$ be defined on all of $X$. It is sufficient to assume they are defined on $A\left(b ; r_{1}\right)$ and that for any $\varepsilon>0$ there exists $A=A(\varepsilon) \in \mathscr{F}(b)$ with $|u(x) / h(x)-l|<\varepsilon$ for $x \in A \cap A\left(b ; r_{1}\right)$.

Let $0<r<r_{1}$ and assume $\left(x_{n}\right) \subset A(b ; r)$ converges to $b$ and that $u\left(x_{n}\right) / h\left(x_{n}\right) \rightarrow$ $l_{1}$. The argument used to prove Theorem 2.2 shows that $l_{1}=l$, i.e., $u / h$ converges through every proper admissible subregion $A(b ; r), 0<r<r_{1}$.

3. The local Fatou theorem. This result is concerned with the admissible convergence of a harmonic function $u$ defined on an open set $U$ which is a union of admissible regions corresponding to a subset of $\bar{N}=\bar{N} \cdot e$ an open dense subset of $K / M$. Here it will be convenient to use the regions $\Gamma^{T}(\bar{n} ; r)$ as the admissible regions.

Following Brelot and Doob [2] one first proves the basic lemma.

Lemma 3.1 (CF. Calderón [3]). Let $E \subset \bar{N}$ be bounded. Assume that to each $\bar{n} \in E$ there is associated a truncated region $\Gamma^{T}(\bar{n} ; r)$ (where $r$ and $T$ may vary with $\bar{n})$ and let $U=\cup_{\bar{n} \in E} \Gamma^{T}(\bar{n} ; r)$.

Let $\varepsilon>0$. Then, for any given $r_{0}>0$, there exists a compact set $D$ and $T_{0}$ such that:

(1) $\cup_{\bar{n} \in D} \Gamma^{T_{0}}\left(\bar{n} ; r_{0}\right) \subset U$; and

(2) the outer measure (relative to dñ) of $E / D$ is $<\varepsilon$.

Proof. A countability argument shows that it suffices to consider the case where $r$ and $T$ are constant.

Sublemma 3.2. Let $\dot{k_{n}} \rightarrow \dot{k}$ and $z \in A^{T}(\dot{k} ; r)=\{k \exp t H \cdot x \mid d(o, x)<r\}$. Then there exists a subsequence $\dot{k}_{n_{1}}$ and $l_{0}=l_{0}(z ; r)$ such that $z \in A^{T}\left(\dot{k_{n}} ; r\right), l>l_{0}$.

Given the sublemma it follows from Lemma 0.4 that there exists a constant $c>1$ such that $\Gamma^{T}\left(\bar{n}_{0} ; r / c\right) \subset U$ if $\bar{n}_{0}$ is in the closure of $E_{0}=\left\{\bar{n} \mid \Gamma^{T}(\bar{n} ; r) \subset U\right\}$. Let $F=\bar{E}_{0}$. 
The Calderón argument used in Lemma 5 of [16] applied to the regions $\Gamma^{T}(\bar{n} ; r / c), \bar{n} \in F$, shows that at each point $\bar{n}_{1}$ of density of $F$ (relative to the translated gauge balls) there exists $T_{0}=T_{0}\left(r_{0}, \bar{n}_{1}\right)$ such that $\Gamma^{T_{0}}\left(\bar{n}_{1} ; r_{0}\right) \subset V=$ $\cup_{n \in F} \Gamma^{T}(\bar{n} ; r / c) \subset U$.

The proof is easily completed by a countability argument since a differentiation theorem holds on $\bar{N}$ for $d \bar{n}$ [17, Corollary 1, p. 394] with the translated gauge balls as a differentiation basis.

Proof OF THE SUblemMA. By compactness there is a subsequence $\left(k_{n_{1}}\right)$ of $\left(k_{n}\right)$ such that $k_{n_{1}} \rightarrow k^{\prime}$ and $\dot{k}^{\prime}=\dot{k}$. Now $z=k^{\prime} \exp t H \cdot x$ with $d(o, x)<r$. Consider $\exp (-t H) k_{n_{l}}^{-1} k^{\prime} \exp t H \cdot x=g_{l} \cdot x=y$. Since $k_{n_{l}} \rightarrow k^{\prime}, g_{l} \rightarrow e$ and so there exists $l_{0}=l_{0}(z, r)$ with $d\left(g_{l} \cdot x, x\right)<r-d(o, x)$ for $l>l_{0}$. Therefore, $d(o, y)<r$ and $k_{n_{l}} \exp t H \cdot y=z$ if $l \geqslant l_{0}$.

COROllary 3.3. For each $r>0, \bar{n} \in E$ implies that there exists $T=T(\bar{n}, r)$ with $\Gamma^{T}(\bar{n}, r) \subset U$ except on a set of d $\bar{n}$-measure zero.

THEOREM 3.4. Let $E \subset \bar{N}$ and assume that for each $\bar{n} \in E$ a truncated region $\Gamma^{T}(\bar{n} ; r)$ is given. Let $U$ be the union of these regions. Then, $U^{c}$ is thin at $\bar{n} \in \bar{E}$, except for a set of dn-measure zero.

Proof. In view of Lemma 3.1 it suffices to assume $E$ is compact and that the parameters $r$ and $T$ are constant, say $r=r_{0}$ and $T=T_{0}$.

Let $u$ be the Poisson integral of $1_{E}$. By Theorem 0.2, $d \bar{n}$-a.e., $u$ converges finely to $1_{E}$. Hence, for $0<\beta<1,\{u \leqslant \beta\}$ is thin $d \bar{n}$-a.e. on $E$. The value of $\beta$ will be chosen below.

Since the complement of a neighbourhood $W$ of $\bar{n}$ is always thin at $\bar{n}$ it suffices to show that $u\left(x_{1}\right)>\beta$ and $x \in W=\left\{n^{\prime} \exp t H \cdot o \mid t \geqslant T_{0}\right\}$ implies $x_{1} \in U$. Let $x_{1}=\bar{n}_{1} \exp t_{1} H \cdot o$. Assume $x_{1} \notin U$. Then $\bar{n}_{1} \cdot b\left(r_{0} e^{-t_{1}}\right) \cap E=\varnothing$, where $b(r)$ is the gauge ball of radius $r$ and centre $e$ in $\bar{N}=\bar{N} \cdot e$. Let $B_{1}=b\left(r_{0} e^{-t_{1}}\right)$ and $B=b\left(r_{0}\right)$. Then, if $F=E^{c}, 1-u\left(x_{1}\right)=\int K(x, \dot{k}) 1_{F}(\dot{k}) d \dot{k} \geqslant \int K(x, \dot{k}) 1_{\bar{n}_{1} \cdot B_{1}}(\dot{k}) d \dot{k}$ $\geqslant c>0$ as on p. 167 of [19]. Consequently, if $\beta>1-c$ it follows that $x_{1} \in U$.

Theorem 3.5 (CF. Carleson [4] ANd Brelot-Doob [2, Théorème 10]). Let $E \subset \bar{N}$ and assume that for each $\bar{n} \in E$ a region $\Gamma^{T}(\bar{n} ; r)$ is given. Let $h$ be a positive harmonic function on $X$ and let $u$ be a harmonic function on $U=\cup_{\bar{n} \in E} \Gamma^{T}(\bar{n} ; r)$. On each $\Gamma^{T}(\bar{n} ; r)$ assume that $u / h$ is either bounded above or below.

Then there is a set $F_{1}$ of $d \bar{n}$-measure zero and a set $F_{2}$ of $\mu_{h}$-measure zero (where $\mu_{h}$ represents $h$ ) such that, for all $\bar{n} \in E \backslash\left(F_{1} \cup F_{2}\right), u / h$ has an admissible limit at $\bar{n}$.

Proof. By a standard device (cf. [2]) it is enough to prove the result for $u>0$ and $U$ connected. Let $F_{1}^{\prime}$ be the subset of $E$ at which $U^{c}$ is not thin, i.e., $F_{1}^{\prime}=\{b \in E \mid U$ is not in $\mathscr{F}(b)\}$.

It follows from the relationship between the Martin boundaries of $U$ and of $X$ (see Theorem A.6 in the Appendix) that $u / h$ has a fine limit at $\mu_{h}$-almost every point of the closure of $E$ at which $U^{c}$ is thin. Let $F_{2}$ be the intersection of this exceptional set with $E$. Then $u / h$ has a fine limit at every point of $E \backslash\left(F_{1}^{\prime} \cup F_{2}\right)$. If $F_{1}$ is the union of $F_{1}^{\prime}$ and the union of the exceptional sets of Corollary 3.3 for an 
increasing sequence $r_{n} \rightarrow+\infty$ it follows from the remark (4) following Theorem 2.2 that $u / h$ has an admissible limit at each point of $E \backslash\left(F_{1} \cup F_{2}\right)$.

4. Admissible convergence implies fine convergence a.e. What follows is a minor adaptation of the proof of Théorème 9 from [2] to the context of a symmetric space $X$.

Let $f: X \rightarrow \mathbf{R}$ and let $E \subset \bar{N}$ be a bounded set of points $\bar{n}$ at which $f(x)$ has a limit as $x \rightarrow \bar{n}, x \in A^{T}(\bar{n} ; r)$ for $r$ fixed. Let $\varphi(\bar{n})$ denote this limit and define $V(T, f)(\bar{n})=\sup \left\{|f(x)-f(y)| \mid x, y \in A^{T}(\bar{n} ; r)\right\}$. It follows from Sublemma 3.2 that $V(T, f)$ is lower semicontinuous and hence $\lim _{T \rightarrow \infty} V(T, f)$ is Borel. This limit is zero on a bounded Borel set $F \supset E$.

By Egorov's theorem there is a compact subset $D_{0}$ of $F$ such that (i) $V(T, f) \rightarrow 0$ uniformly on $D_{0}$ and (ii) the $d \bar{n}$ measure of $F \backslash D_{0}$ is $<\varepsilon$.

Let $\delta>0$. Choose $T=T(\delta)$ such that $V(T, f)<\delta$ on $D_{0}$. Let $U(\delta)=$ $\cup_{\bar{n} \in D_{0} \cap E} A^{T}(\bar{n} ; r)$. This is an open set and by Lemma 0.4 and Theorem 3.4 is in $\mathscr{F}(\bar{n})$ for $d \bar{n}$-almost all $\bar{n} \in D_{0} \cap E$.

Lemma 0.4 and the fact that $V(T, f) \rightarrow 0$ uniformly on $D_{0}$ implies that $\varphi$ is continuous on $D_{0} \cap E$. Let $y \in A^{T}(\bar{n} ; r)$ and $\bar{n}, \bar{n}_{0} \in D_{0} \cap E$. Then $\left|f(y)-\varphi\left(\bar{n}_{0}\right)\right|$ $<|f(y)-\varphi(\bar{n})|+\left|\varphi(\bar{n})-\varphi\left(\bar{n}_{0}\right)\right| \leqslant \delta+\left|\varphi(\bar{n})-\varphi\left(\bar{n}_{0}\right)\right|<2 \delta$ if $\bar{n}$ is close enough to $\bar{n}_{0}$.

In other words there is a neighbourhood $W$ of $\bar{n}_{0}$ such that $U(\delta) \cap W \subset\{y \in$ $\left.X|| f(y)-\varphi\left(\bar{n}_{0}\right) \mid \leqslant 2 \delta\right\}$. If $U(\delta) \in \mathscr{F}\left(\bar{n}_{0}\right)$ for all $\delta$ then $f$ converges finely at $\bar{n}_{0}$ to $\varphi\left(\bar{n}_{0}\right)$. As this hypothesis holds for $d \bar{n}$-almost all points of $D_{0} \cap E$ it follows that $d \bar{n}$-a.e. on $F$ the function $f$ has a fine limit at $\bar{n}$ equal to $\varphi(\bar{n})$. This completes the proof of the following result.

THEOREM 4.1. Let $f: X \rightarrow \mathbf{R}$ and let $E(r) \subset \bar{N}$ be the set of points $\bar{n}$ at which $f(x)$ has a limit $\varphi(\bar{n})$ as $x \rightarrow \bar{n}, x \in A^{T}(\bar{n} ; r)$ for some fixed $r>0$. Then $E(r)$ is a Borel set and, at d $\bar{n}$-almost every point $\bar{n}$ of $E(r), f$ converges finely to $\varphi(\bar{n})$.

In particular, if $E \subset \bar{N}$ is the set of points $\bar{n}$ at which f has an admissible limit $\varphi(\bar{n})$ then it is Borel and at din-almost every point of $E$ the function $f$ converges finely to $\varphi(\bar{n})$.

Appendix. The relation between the Martin boundaries of $X$ and of a connected open subset $W$.

1. In the third paragraph of the proof of Théorème 10 in [2] Brelot and Doob state (for the half-space) the result to be presented below as Proposition A.5. While for the purposes of this article $X$ is a symmetric space and the potential theory is associated with $L u=\Delta u-c u$, the arguments below apply in a larger context (namely, an axiomatic potential theory in the sense of Brelot for which the so-called axiom D is satisfied [14]). It is also quite likely that Proposition A.5 holds in the context of probabilistic potential theory.

The result in question is the following: let $h>0$ be harmonic on $X$ and let $h^{\prime}=\left.h\right|_{W}$; on the "intersection" of the Martin boundaries of $X$ and $W$ the measures representing $h$ and $h^{\prime}$ respectively are mutually absolutely continuous. In particular, if $h=1$, the harmonic measures associated with the two Martin compactifications 
are mutually absolutely continuous on the common boundary. In [2] this result is attributed to Naïm [27, Lemme 6, p. 247]. However, Naïm's result is not the same.

2. To begin with, the following result of Brelot shows that a subset of the Martin boundary of $X$ can be identified with a subset of the Martin boundary of $W$.

Theorem A.1 (Brelot, Théorème 12, P. 220 [22]). Let $\Delta_{1}(X, W)=\{b \in$ $\Delta_{1}(x) \mid W^{c}$ is thin at $\left.b\right\}$. Then for each $b \in \Delta_{1}(X, W)$ the function $K_{b}-\hat{R}_{W^{c}} K_{b}=k_{b}$ is a minimal harmonic function on $W$. Further, the map $K_{b} \leadsto k_{b}$ is injective.

Proof. The argument given in [22] applies without change. For the reader's convenience it is outlined below.

Let $h=K_{b}$ and assume $0 \leqslant k \leqslant h-\hat{R}_{W^{c}} h$ on $W$. Then there is a superharmonic function $w$ on $X$ such that (i) $w(x)=k(x)+\hat{R}_{W^{c}} h(x)$ for $x \in W$ and (ii) except for a polar subset of $\partial W, w(x)=h(x)$ for $x \in W^{c}$. Clearly, $w<h$.

Let $p+l=w$ be the Riesz decomposition of $w$ with $l$ harmonic. Then $l=c h$, $0 \leqslant c \leqslant 1$. Because the exceptional set in (ii) is polar $\hat{R}_{W^{c} w}=\hat{R}_{W^{c}} h$. Hence, $\hat{R}_{W^{c}} p=(1-c) \hat{R}_{W^{c}} h$.

Since $p \leqslant h$ the potential $p=G \mu$ is locally bounded. Consequently, $\mu$ does not charge any polar set. From this it follows that $\hat{R}_{W^{c}} p=p$ (by using the fact that polar and copolar sets are the same).

Hence, $p=(1-c) \hat{R}_{W^{c}} h$. On $W$ it follows that $k=w-\hat{R}_{W^{c}} h=p+c h-\hat{R}_{W^{c}} h$ $=c\left(h-\hat{R}_{W^{c}} h\right)$.

The injectivity of the map $K_{b} \leadsto k_{b}$ follows from the uniqueness of the Riesz decomposition: $h_{i}=K_{b_{i}}$ and $k_{b_{1}}=k_{b_{2}}$ implies $h_{1}+\hat{R}_{W^{c}} h_{2}=h_{2}+\hat{R}_{W^{c}} h_{1}$ on $X$ (except for a polar set, and hence everywhere); $\hat{R}_{W^{c}} h_{i}$ is a potential and so $h_{1}=h_{2}$.

3. Define $B: \Delta_{1}(X, W) \rightarrow \Delta_{1}(W)$ by setting $B(b)=\bar{b}$ if $\bar{b}$ corresponds to $K_{b}-$ $\hat{R}_{W^{c}} K_{b}=k_{b}$. The kernel $K(x, b)$ may be assumed to be normalized at a point $a \in W$. Using the same point for the corresponding kernel $\bar{k}$ on $W$ it follows that $\bar{k}(x, \bar{b})\left[1-\hat{R}_{W^{c}} K_{b}(a)\right]=K(x, b)-\hat{R}_{W^{c}} K_{b}(x)=k_{b}(x)$.

The next step is to show that $\Delta_{1}(X, W)$ and $B\left(\Delta_{1}(X, W)\right)$ are both Borel subsets of $\Delta_{1}(X)$ (resp. $\Delta_{1}(W)$ ). To this end set $\Delta_{1}(X, W ; n)=\left\{b \in \Delta_{1}(X, W) \mid 1-\right.$ $\left.\hat{R}_{W^{c}} K_{b}(a) \geqslant 1 / n\right\}$. It clearly suffices to show that, for each $n \geqslant 1, \Delta_{1}(X, W ; n)$ and $B\left(\Delta_{1}(X, W ; n)\right)$ are Borel subsets of $\Delta_{1}(X)$ (resp. $\left.\Delta_{1}(W)\right)$.

Proposition A.2. For each $n \geqslant 1, \Delta_{1}(X, W ; n)$ is a Borel subset of $\Delta_{1}(X)$ and $B$ restricted to this subset is a continuous injection.

Proof. Fatou's lemma implies that $b \leadsto 1-\hat{R}_{W^{c}} K_{b}(a)$ is upper semicontinuous. Hence, $\Delta_{1}(X, W ; n)$ is a closed subset of $\Delta_{1}(X)$.

Assume $\left(b_{m}\right) \subset \Delta_{1}(X, W ; n)$ converges to $b \in \Delta_{1}(X, W ; n)$. Let $k_{m}(x)=K_{m}(x)$ - $\hat{R}_{W^{c}} K_{m}(x)$, where $K_{m}(x)=K\left(x, b_{m}\right)$. Since $1>k_{m}(a) \geqslant 1 / n$, a subsequence $k_{m}$ converges to a harmonic function $l$ on $W$. Further, $K\left(x, b_{m}\right) \rightarrow K(x, b)$ and so by Fatou's lemma, $\hat{R}_{W^{c}} K_{b} \leqslant \lim _{m} R_{W^{c}} K_{m}$.

Consequently, $l \leqslant k_{b}=K_{b}-\hat{R}_{W^{c}} K_{b}$. Hence, $l=c \bar{k}_{b}$ with $c=l(a)$. Hence, $\left[1 / k_{m}(a)\right] k_{m}=\bar{k}_{b_{m}} \rightarrow \bar{k}_{b}$.

Corollary A.3. For each $n, B\left(\Delta_{1}(X, W ; n)\right)$ is a Borel subset of $\Delta_{1}(W)$. 
Proof. The Martin boundary $\Delta(X)$ of $X$ is a complete separable metric space (i.e. a Polish space in the terminology of [1]). By Théorème 3 on p. 134 of [1], $\Delta_{1}(X, W ; n)$ is a Lusin subspace of $\Delta(X)$. The corollary to Lemma 7 on p. 135 of [1] implies the desired result.

REMARK. In case $\Delta_{1}(X)$ is compact this result is obvious since $\Delta_{1}(X, W ; n)$ is compact for each $n$. This is the case for symmetric spaces of rank one.

Corollary A.4. $B: \Delta_{1}(X, W) \rightarrow \Delta_{1}(W)$ is a Borel injection (and even a Borel isomorphism with the image).

4. Let $h>0$ be harmonic on $X$ and have representing measure $\mu$ on $\Delta_{1}(X)$. Let $\nu$ be the representing measure on $\Delta_{1}(W)$ for $h^{\prime}=\left.h\right|_{W}$.

Proposition A.5. Let $\Gamma \subset \Delta_{1}(X, W)$ be Borel and let $B(\Gamma)=\Lambda$. Then

$$
\nu(\Lambda)=\int_{\Gamma}\left\{1-\hat{R}_{W^{c}} K_{b}(a)\right\} \mu(d b) .
$$

Hence, $\nu(\Lambda)=0 \Leftrightarrow \mu(\Gamma)=0$.

Proof. Let $h_{1}(x)=\int_{\Delta_{1}(X, W)} K(x, b) \mu(d b)$. Then $h=h_{1}+h_{2}$, where $\hat{R}_{W^{c}} h_{2}=h_{2}$. Further,

$$
\begin{aligned}
h_{1}(x) & =\int_{\Delta_{1}(X, W)}\left\{K(x, b)-\hat{R}_{W^{c}} K_{b}(x)\right\} \mu(d b)+\int_{\Delta_{1}(X, W)} \hat{R}_{W^{c}} K_{b}(x) \mu(d b) \\
& =k(x)+p(x),
\end{aligned}
$$

where $p(x)$ is a potential on $X$ (as each $\hat{R}_{W^{c}} K_{b}$ is a potential).

Let $\omega(\Lambda)=\int_{\Gamma}\left\{1-\hat{R}_{W^{c}} K_{b}(a)\right\} \mu d b$, if $B(\Gamma)=\Lambda$. Then $\left.k\right|_{W}=\int \bar{k}(, \bar{b}) \omega(d \bar{b})$. Since $\nu \geqslant \omega$, to establish (*) it suffices to show that the representing measure on $\Delta_{1}(W)$ for any positive harmonic function $u<\left.\left(p+h_{2}\right)\right|_{W}$ has no mass in $B\left(\Delta_{1}(X, W)\right)$ as this will imply that the measure $\nu-\omega$ does not charge $B\left(\Delta_{1}(X, W)\right)$.

Let $u(x)=\int \bar{k}(x, \bar{b}) \eta(d \bar{b}) \leqslant p(x)+h_{2}(x)$ for all $x \in W$, where $\eta$ is a positive measure on $B\left(\Delta_{1}(X, W)\right)$. Then there is a unique measure $\lambda$ on $\Delta_{1}(X, W)$ related to $\eta$ by the formula (*). This measure $\lambda$ is such that

$$
\int\left\{K(x, b)-\hat{R}_{W^{c}} K_{b}(x)\right\} \lambda(d b)=u(x) \text { for all } x \in W .
$$

Hence, $w(x)=\int K(x, b) \lambda(d b) \leqslant p_{1}(x)+p(x)+h_{2}(x)$, where $p_{1}=\hat{R}_{W^{c} w}$, for all $x \in W$. Consequently, $w \leqslant p_{1}+p+h_{2}$ on $X$ since the possible exceptional set is polar. Since $p_{1}+p$ is a potential, $w \leqslant h_{2}$. Now the representing measure for $h_{2}$ does not charge $\Delta_{1}(X, W)$ and so consequently $\lambda=0$. Hence, $\eta=0$.

5. TheOREM A.6. Let $h>0$ be harmonic on $X$ and $u>0$ harmonic on $W$. Then $u / h$ has a fine limit at $b \in \Delta_{1}(X, W)$ if and only if $u / h$ has a fine limit on $W$ at $\bar{b}=B(b) \in B\left(\Delta_{1}(X, W)\right)$. Further, if $E$ is the exceptional subset of $\Delta_{1}(W)$ where $u / h$ has no fine limit on $W$, then $\mu_{h}\left(B^{-1}(E)\right)=0$ (where $\mu_{h}$ is the representing measure for $h$ ).

Proof. The second statement follows immediately from Proposition A.5. 
For all $b \in \Delta_{1}(X, W), W \in \mathscr{F}(b)$. Hence it suffices to show that $A \subset W$ is in the fine filter on $W$ at $\bar{b}$ if and only if $A=A_{1} \cap W$, with $A_{1} \in \mathcal{F}(b)$ : in other words, $E \subset W$ is thin at $\bar{b}$ (on $W$ ) if and only if $E=E_{1} \cap W$ where $E_{1}$ is thin at $b$.

This follows from the next lemma with $u=K_{b}$.

LeMmA A.7 (CF. LEMME 5, P. 223 [22]). Let $u \geqslant 0$ be superharmonic on $X$ and let $A \subset W$. Then, on $W,\left(\hat{R}^{W}\right)_{A}\left(u-\hat{R}_{W^{c}} u\right)=\hat{R}_{\left(A \cup W^{c}\right)} u-\hat{R}_{W^{c}} u$, where $\left(\hat{R}^{W}\right)_{A}$ is the operator that performs "balayage onto $A$ " in the subspace $W$.

Proof. Let $v$ be superharmonic on $X$ with $v \geqslant u$ on $A \cup W^{c}$. Then $\hat{R}_{W^{c} v} \geqslant$ $\hat{R}_{W^{c}} u$ and so $v-\hat{R}_{W^{c}} u \geqslant 0$ and is $\geqslant u-\hat{R}_{W^{c}} u$ on $A$. Hence, on $W$,

$$
\left(\hat{R}^{W}\right)_{A}\left(u-\hat{R}_{W^{c} u} u\right) \leqslant \hat{R}_{A \cup W^{c} u}-\hat{R}_{W^{c} u} .
$$

Conversely, if $u-\hat{R}_{W^{c}} u \geqslant w$ and $w \geqslant u-\hat{R}_{W^{c} u}$ on $A$ (where $w$ is superharmonic on $W$ ) there is a superharmonic function $v$ on $X$ whose restriction to $W$ is $w+\hat{R}_{W^{c}} u$ (cf. proof of Theorem A.1) and which agrees with $u$ on the set $B$ of regular points of $W^{c}$. Hence, $v \geqslant u$ on $A \cup B$.

Therefore, $\left(R^{W}\right)_{A}\left(u-\hat{R}_{W^{c}} u\right)+\hat{R}_{W^{c}} u \geqslant R_{A \cup B} u$ on $W$. Consequently, on $W$,

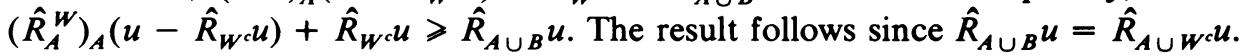

\section{BIBLIOGRAPHY}

1. N. Bourbaki, Eléménts de mathématiques. Topologie générale, Chap. 9, $2^{\mathrm{e}}$ ed., Hermann, Paris, 1958.

2. M. Brelot and J. L. Doob, Limites angulaires et limites fines, Ann. Inst. Fourier (Grenoble) 13 (2) (1963), 395-415.

3. A. P. Calderon, On the behaviour of harmonic functions at the boundary, Trans. Amer. Math. Soc. 68 (1950), 47-54.

4. L. Carleson, On the existence of boundary values for harmonic functions in several variables, Ark. Mat. 4 (1961), 393-399.

5. E. A. Coddington and N. Levinson, Theory of ordinary differential equations, McGraw-Hill, New York, 1955.

6. R. Courant and D. Hilbert, Methods of mathematical physics. II, Interscience, New York, 1962.

7. A. Debiard, Comparison des espaces $H^{p}$ géométrique et probabilistes au dessus de l'espace hermitien hyperbolique, Bull. Sci. Math. 103 (1979), 305-351.

8. J. L. Doob, Conditional Brownian motion and the boundary limits of harmonic functions, Bull. Soc. Math. France 85 (1957), 431-458.

9. A non-probabilistic proof of the relative Fatou theorem, Ann. Inst. Fourier (Grenoble) 9 (1959), 293-300.

10. M. Flensted-Jensen, Paley-Wiener type theorems for a differential operator connected with symmetric spaces, Ark. Mat. 10 (1972), 143-162.

11. H. Föllmer, Feine Topologie am Martinrand eines Standardprozesses, Z. Wahrsch. Verw. Gebiete 12 (1969), 127-144.

12. K. Gowrisankaran, Extremal harmonic functions and boundary value problems, Ann. Inst. Fourier (Grenoble) 13 (2) (1963), 307-356.

13. S. Helgason, Differential geometry and symmetric spaces, Academic Press, New York, 1969.

14. Mme. R. M. Hervé, Recherches axiomatiques sur la théorie des fonctions surharmoniques et du potentiel, Ann. Inst. Fourier (Grenoble) 12 (1962), 415-571.

15. F. I. Karpelevic, The geometry of geodesics and the eigenfunctions of the Beltrami-Laplace operator on symmetric spaces, Trans. Moscow Math. Soc. 14 (1965), 51-199.

16. A. Korányi, Harmonic functions on hermitian hyperbolic space, Trans. Amer. Math. Soc. 135 (1969), 507-516.

17. _ Harmonic functions on symmetric spaces, Symmetric Spaces (W. M. Boothby and G. Weiss, eds.), Marcel Dekker, New York, 1972. 
18. A survey of harmonic functions on symmetric spaces, Proc. Sympos. Pure Math., vol. 35, Amer. Math. Soc., Providence, R. I., 1979.

19. A. Koranyi and R. B. Putz, Local Fatou theorem and area theorem for symmetric spaces of rank one, Trans. Amer. Math. Soc. 224 (1976), 157-168.

20. R. S. Martin, Minimal positive harmonic functions, Trans. Amer. Math. Soc. 49 (1941), 137-172.

21. H. L. Michelson, Fatou theorems for eigenfunctions of the invariant differential operators on symmetric spaces, Trans. Amer. Math. Soc. 177 (1973), 257-274.

22. L. Naïm, Sur le role de la frontière de R. S. Martin dans la théorie du potentiel, Ann. Inst. Fourier (Grenoble) 7 (1957), 183-281.

23. J. Serrin, On the Harnack inequality for linear elliptic equations, J. Analyse Math. 4 (1955/56), 292-308.

24. J. C. Taylor, Martin boundaries of equivalent sheaves, Ann. Inst. Fourier (Grenoble) 20 (1970), 433-456.

25. Fine and admissible convergence for the unit ball in $C^{n}$ (Proc. 1979 Copenhagen Potential Theory Colloquium, 1979), Lecture Notes in Math., vol. 787, Springer-Verlag, Berlin and New York (to appear).

26. G. Warner, Harmonic analysis on semi-simple Lie groups. I, Die Grundlehren der Math. Wissenschaften, Band 188, Springer-Verlag, Berlin, 1972.

Department of Mathematics, Washington University, St. Louis, Missouri 63130

Department of Mathematics, McGill University, 805 Sherbrooke Street West, Montreal, Quebec, Canada H3A 2K6 